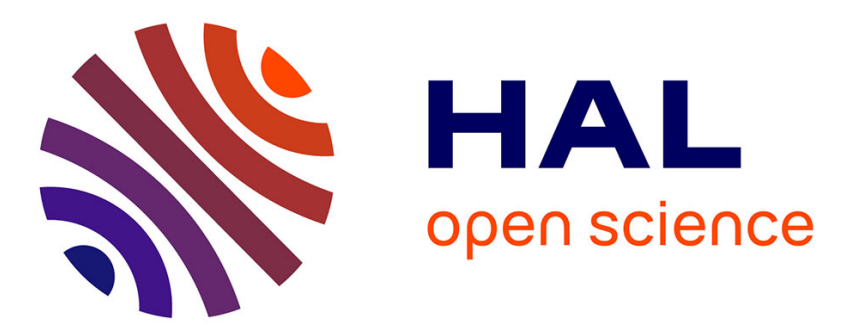

\title{
Comment devient-on Indigène ou Chérif par une origine juive? Trajectoires généalogiques pour repenser le lien au sol en Amazonie et au Maroc Romain Simenel, Émilie Stoll
}

\section{To cite this version:}

Romain Simenel, Émilie Stoll. Comment devient-on Indigène ou Chérif par une origine juive? Trajectoires généalogiques pour repenser le lien au sol en Amazonie et au Maroc. Lusotopie, 2020, Judaísmos nas lusotopias contemporâneas, 18 (2), pp.218-246. 10.1163/17683084-12341744 . hal-02482248

\author{
HAL Id: hal-02482248 \\ https://hal.science/hal-02482248
}

Submitted on 17 Feb 2020

HAL is a multi-disciplinary open access archive for the deposit and dissemination of scientific research documents, whether they are published or not. The documents may come from teaching and research institutions in France or abroad, or from public or private research centers.
L'archive ouverte pluridisciplinaire HAL, est destinée au dépôt et à la diffusion de documents scientifiques de niveau recherche, publiés ou non, émanant des établissements d'enseignement et de recherche français ou étrangers, des laboratoires publics ou privés. 


\title{
Comment devient-on Indigène ou Chérif par une origine juive ? \\ Trajectoires généalogiques pour repenser le lien au sol en Amazonie et au Maroc
}

Como identificar-se indígena ou xerife com uma origem judaica?

Trajetórias genealógica para repensar o vínculo com a terra na Amazônia e no Marrocos

Claiming to be Indigenous or Sharif with a Jewish origin?

Genealogical trajectories to rethink land connection in the Amazon and Morocco

\author{
Romain Simenel $^{1}$ \\ Emilie Stoll ${ }^{2}$
}

\section{Résumé :}

Quel est le point commun entre un chérif musulman (descendant du prophète Mohammed) de la confédération Aït Ba'amran des tribus du Sud-Ouest marocain et un Indigène animiste d'Amazonie brésilienne ? À la surprise de l'ethnologue, tous deux sont affiliés à une ascendance juive. Cette judaïté, conçue davantage comme une origine que comme une pratique religieuse, participe à préciser le lien au sol des générations actuelles. La mise en regard de deux cas ethnographiques permet une discussion plus large sur la multiplicité des origines en contexte migratoire dans le temps long. La judaïté des origines participe à asseoir pleinement l'inscription actuelle des populations au sein d'un espace marqué par l'histoire de la construction des Etats empires portugais et espagnols.

Mots-clés : Identification - Immigration juive - Lien au sol - post migration - Amazonie - Maroc Empire portugais

\section{Resumo:}

O que têm em comum um xerife muçulmano (descendente do profeta Mohammed) da confederação Ait Baamrane das tribos do sudoeste do Marrocos e um indígena animista da Amazônia brasileira? Para surpresa do etnólogo, ambos reclamam uma ascendência hebraica. Esse judaísmo, concebido mais como origem do que como prática religiosa, ajuda esclarecer o vínculo com a terra das gerações presentes. A comparação desses dois casos etnográficos proporciona uma discussão mais ampla sobre a multiplicidade de origens em um contexto migratório de longo prazo. A origem hebraica contribui então para legitimar a inscrição atual das populações dentro de um espaço marcado pela história das colonizações portuguesa e espanhola.

Palavras chaves: Identificação - Imigração judaica - vínculo com a terra - pós migração - Amazônia - Marrocos- Império português

\footnotetext{
Abstract:

What is the common point between a Muslim Sharif (a descendant of Prophet Mohammed) from the Ait Baamrane confederation of the tribes of southwestern Morocco and an Indigenous person from

${ }^{1}$ romain.simenel@ird.fr chargé de recherche à l'IRD, Muséum National d'Histoire Naturelle - IRD / UMR 208 Patrimoines Locaux, Environnement e Globalisation (PALOC)

2 emilie.stoll@ cnrs.fr chargée de recherche au CNRS, Université de Paris - Université Côte d'Azur - CNRS IRD / Unité de Recherche Migrations et Société (URMIS)
} 
the animist Amazon, in Brazil? To the ethnologist's surprise, both are affiliated to a Jewish ancestry. And this Judaism, conceived more as an origin than as a religious practice, helps to clarify the link to the land of the present generations. The comparison of these two ethnographic cases allows a wider discussion on the multiplicity of origins in a long-term migratory context. The Jewishness of the origins helps to fully establish the current inscription of the populations within a space marked by the history of Portuguese and Spanish colonization.

Key words: Identification - Jewish diaspora - land connection - postmigration - Amazonia - Morocco - Portuguese Empire

\section{Remerciements}

Les enquêtes de terrain et la rédaction de cet article ont été financées par les projets suivants: Emergence(s) Ville de Paris EXORIGINS De la diversité bio-culturelle dans les jardins des Parisiens Circulations croisées de végétaux, de personnes et d'imaginaires, H2020 MSCA RISE 2015 nº61053 ODYSSEA Observatory of the dynamics of interactions between societies and environment in the Amazon, CAPES-Cofecub Sh904-18 IBIS Intégration, biodiversité et socio-écologie - l'utilisation des sols dans des territoires amazoniens et caribéens, CAPES-Cofecub Sh811-14 Reconfigurations foncières et réélaborations identitaires en Amazonie brésilienne. 


\section{Introduction}

Quel est le lien entre le Portugal, un chérif musulman (descendant du prophète Mohammed) de la confédération Aït Ba'amran des tribus du Sud-Ouest marocain et un indigène (indígena $)^{3}$ animiste d'Amazonie brésilienne ? Cet article propose un éclairage sur les processus d'intégration de l'étranger, à partir d'exemples d'individus qui s'inscrivent dans des circuits migratoires où le Maroc est un lieu charnière de la colonisation d'origine portugaise vers le Nouveau Monde. Plus spécifiquement, nous explorerons la circulation d'un collectif singulier - les Juifs (convertis ou non) - entre Portugal, Maroc et Brésil. À partir de deux trajectoires familiales paradigmatiques, nous analyserons la façon dont des individus identifiés comme « Juifs » sont inscrits dans les généalogies locales, favorisant un processus d'ancrage social et territorial. Dans ces trajectoires, la confession juive disparaît au profit de discours sur l'origine, permettant la transmission de compétences et savoirs singuliers.

Contrairement à une idée-reçue, le judaïsme est la confession monothéiste qui s'est mélangée aux autres cultures le plus tôt dans l'histoire, résultat des migrations répétées des Juifs dans le temps et l'espace. Des Falachas d'Ethiopie aux Juifs Kéralais en passant par les Igbobenei-Israël du Nigéria ou encore les Juifs berbères du Maroc, de nombreux collectifs se réclament d'une confession juive historique structurante de leur identité. Plus que la confession, l'origine juive a ainsi une propension à être revendiquée par des groupes de cultures très différentes.

Les circulations des populations juives et les récits sur leurs origines ont suivi des trajectoires de mondialisation différentes parmi lesquelles celles impulsées par la construction des futurs états ibériques, espagnol et portugais. Dans le sillage de la conquête catholique de l'Andalousie par les Espagnols (IX-X ${ }^{\mathrm{e}} \mathrm{s}$.), puis par les Portugais (XII ${ }^{\mathrm{e}} \mathrm{s}$.), de nombreux Juifs s'exilent au Maroc. Connu pour avoir été sous domination espagnole et française, le Maroc a également abrité des comptoirs commerciaux portugais le long de ses côtes, du $\mathrm{XV}^{\mathrm{e}} \mathrm{s}$. au XVIII ${ }^{\mathrm{e}}$ s. - de Ceuta à Bojador (Boujdour), incluant Mogador (Essaouira), Anfa (Casablanca), Tanger, parmi d'autres - jusqu'à la reddition de la place forte de Mazagan (El Jadida), en 1769. À partir de ces comptoirs, des flux de populations d'origine portugaise sont partis en Amérique du Sud dès la conquête du Nouveau Monde (XV-XIX ${ }^{\mathrm{e}} \mathrm{s}$.), motivés par le commerce des épices, du cacao et, plus tardivement, par le boom du caoutchouc $\left(\mathrm{XIX}^{\mathrm{e}} \mathrm{s}\right)$. Dans les réseaux portugais,

\footnotetext{
${ }^{3}$ Terme auto-référent utilisé en Amazonie brésilienne par ceux qui s’identifient politiquement à la catégorie légale Indigène.
} 
l'exemple le plus emblématique est celui des habitants de la ville coloniale de Mazagan, entièrement déplacée du Maroc vers l'Amazonie sur ordre du Roi (Vidal 2005).

Le propos de cet article est de situer la place de l'origine juive dans les stratégies d'intégration de migrants issus des trajectoires de mondialisation impulsées par la construction des Etats Empires espagnol et portugais au Maroc et au Brésil, et son rôle dans l'identification sociale actuelle de leurs descendants. Exil contraint ou opportunités économiques puis sociales, les raisons de la migration des Juifs sont plurielles et s'accumulent dans l'histoire de ces familles. Nous partirons d'une comparaison entre deux destinées, en montrant comment l'origine juive sert des collectifs amérindiens au Brésil, ou des lignées de descendants du prophète Mohammed au Maroc, pour légitimer le lien au sol dans un univers cosmopolite et se positionner vis à vis des identités englobantes, religieuses ou politiques. À la mobilité historique des populations juives sur la planète, s'ajoute donc la circulation de leurs origines dans les généalogies de peuples qualifiés d'autochtones comme les «peuples indigènes » d'Amazonie ou les Berbères (Imazighen selon les militants) du Maroc. Reste à savoir si la seconde est la conséquence de la première, un peu comme si la répétition du phénomène migratoire dans le temps et dans l'espace aurait fini par induire l'omniprésence de l'origine exogène - ici juive dans la référence à l'altérité généalogique dans de nombreuses cultures du monde.

L'analyse des systèmes de parenté présentée dans la partie marocaine résulte du terrain de thèse de Romain Simenel, mené de 2002 à 2004 dans un village, par insertion de l'ethnographe au sein d'une famille. Les données amazoniennes ont été collectées par Emilie Stoll dans le cadre d'une enquête ethnographique initiée en 2011 et toujours en cours, entre cinq villages de la commune de Santarém.

\section{L'origine juive dans la construction du chérifisme dans le Sud du Maroc}

\subsection{Le Maroc et les Juifs : histoires d'une terre d'accueil}

L'histoire des Juifs au Maroc ne demande qu'à être écrite encore et toujours tant les archives et les mémoires orales abondent à son sujet et ce même si certains historiens en ont déjà écrit les pages principales, à l'instar de Mohammed Kenbib (1994) ou d'André Levy (2003). Selon Jacques-Meunié, les premières populations juives du Maroc vinrent par la mer, dans la région sud-ouest, plus précisément au Souss et au nord du Sahara, à l'époque de la navigation phénicienne pour des raisons marchandes. Puis, dans un deuxième temps, elles arrivèrent par la terre, après la déportation des dix tribus d'Israël au VIII ${ }^{\mathrm{e}}$ siècle et à la suite de la première destruction du temple de Jérusalem au $\mathrm{VI}^{\mathrm{e}}$ siècle avant $\mathrm{J}-\mathrm{C}$ pour y trouver refuge (1982: 61). La tradition orale en tachelhit (langue berbère locale) continue d'entretenir la 
mémoire d'une ancienne présence juive, au point de qualifier le Souss de lhebss $n$ Seidna Suleyman, la «prison du prophète Salomon », en référence aux tribus juives que ce dernier aurait envoyé en exil en ce lieu (Simenel 2010).

Selon les termes de Jacques-Meunié, des « royaumes juifs » auraient vu le jour dès le $\mathrm{VI}^{\mathrm{e}}$ siècle avant J-C, tels le Royaume d'Ifrane puis celui du Dra'a (1982 : 61-62). L'afflux de groupes ou d'individus de confession juive au Maroc a perduré au fil des siècles suivant, notamment après le début de la conquête de l'Andalousie musulmane par les espagnols puis par les portugais et l'arrivée de Juifs andalous. Si la plupart des grandes villes du Souss comme Tiznit ou Guelmim (Figure 1) disposait d'un quartier juif, le mellah, la majeure partie des communautés juives habitait le monde rural, en témoignent encore les cimetières hébraïques, seuls vestiges de cette ancienne occupation.

Figure 1. Localisation des lieux de l'étude au Maroc

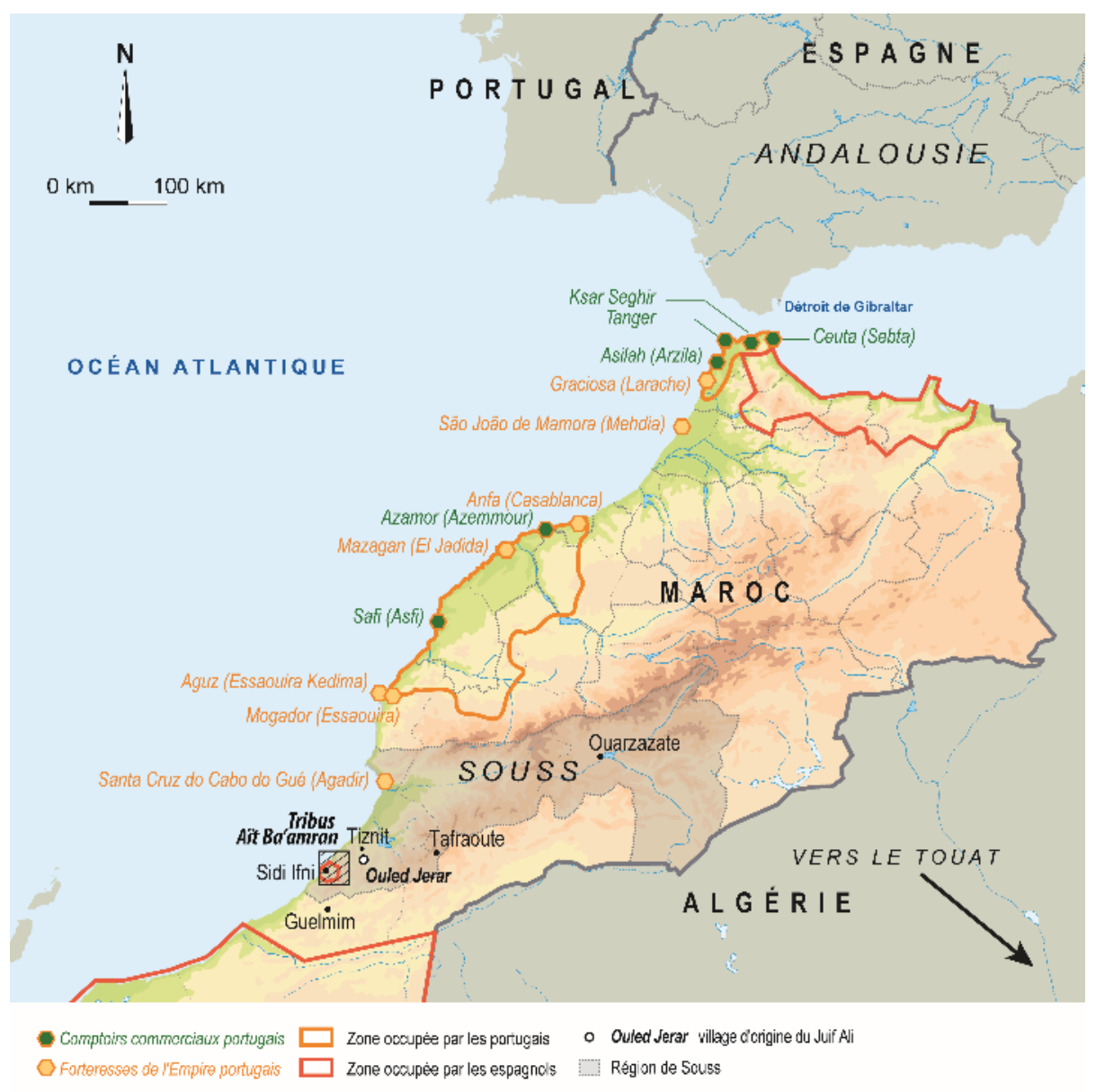

Réalisation : Laurence Billault - IRD. 
Aujourd'hui, hormis une poignée d'individus, les Juifs ont quitté le Souss, mais ils restent néanmoins une référence incontournable dans la tradition orale et la mémoire collective sur le peuplement de la région. Ainsi, de nombreux récits oraux sur la sainteté font référence à des personnages juifs. Dans le domaine ésotérique, la « magie juive » (siher n ouday) est considérée comme la plus redoutable. Le souvenir de la présence des Juifs est entretenu dans le paysage par le biais de la toponymie ; on ne compte plus les villages berbères dont les noms ont des consonances hébraïques tels que Id Dib, Id Soussan, Id Daoud, etc. Le souvenir de la présence juive et de ses origines est toujours vivace dans la mémoire des habitants. Les anciennes communautés juives localisées à l'intérieur des terres seraient initialement venues de la région du Touat (ouest du Sahara algérien), tandis que celles situées sur la côte atlantique, comme Oulad Jerar, seraient arrivées du Portugal. Mais c'est dans les généalogies que la référence aux Juifs reste la plus vivace, et plus particulièrement dans les généalogies de chorfa (sing. chérif), ces descendants du prophète Mohammed dont le rôle est de répandre la bénédiction divine (baraka) et de réguler les conflits entre tribus, fractions ou lignages (Jamous 1981). Si l'on sait que de nombreux Juifs marocains se sont convertis à l'Islam, on ignore souvent leur parcours, la manière dont ils ont été intégrés aux communautés musulmanes et sous quel rang social.

\subsection{Les Juifs dans le système d'intégration d'étrangers au Maroc}

Pour comprendre comment des Juifs ont pu être intégrés à des lignages musulmans au Maroc, encore faut-il saisir le mécanisme de la circulation des hommes et son pendant, l'intégration d'étrangers, dans la logique de reproduction des groupes sociaux. Dès le XIV siècle, l'historien Ibn Khaldoun considère l'exil masculin comme un trait saillant des sociétés du Maghreb et qualifie le processus fréquent d'intégration d'étrangers au sein des lignages de « secret de Dieu » (1967 : 202-203). Au Maroc, depuis des siècles, le bannissement des hommes est une institution juridique régulée par le droit coutumier. En cas de crime, de vol, de viol, d'adultère ou même pour des motifs de rivalité politique ou religieuse, les hommes désignés coupables pouvaient être expulsés de leur lignage et de leur tribu et condamnés à prendre la route de l'exil en quête d'une terre d'accueil (Gellner 2003 : 129, Sebti 1992 : 62, Berque 1955). Le mécanisme du bannissement évite une escalade de la violence entre les groupes concernés. Une fois chassé de son lignage, le banni (amzouag) est dépourvu de lien familial et donc de droit d'héritage. Il est vierge de toute filiation et son objectif principal, pour continuer d'exister socialement, est de trouver dans une autre tribu une femme à épouser. Le pays des tribus Aït Ba'amran, situé dans le sud-ouest du Souss, est depuis longtemps réputé dans tout le Maroc pour être une terre d'exil (Simenel 2010). Tout étranger qui cherche à s'installer durablement 
en pays Aït Ba'amran est considéré comme un banni. Selon l'histoire locale, les Juifs seraient les premiers bannis à être arrivés en ces terres. Mais quelle est donc la place du banni et, qui plus est du banni juif, dans la dynamique sociale et historique des lignages ?

Dans les régions du sud-ouest du Maroc, un patrilignage perdure comme unité spatiale et historique tant que son patrimoine résidentiel et foncier reste indivis dans le giron de sa descendance mâle. Le temps passant et les générations se multipliant, il arrive que les terres d'un lignage, et plus rarement sa résidence, tombent en grande partie entre les mains des femmes. Les situations les plus fréquentes de risque d'appropriation du patrimoine résidentiel ou terrien d'un patrilignage par les femmes sont l'absence de descendance masculine (femmes sans frère) et le veuvage (du point de vue du lignage du défunt). Le mariage de l'héritière avec un homme d'un lignage voisin ou d'une autre tribu constitue un grand risque d'éclatement du patrimoine lignager entre affins. Pour reproduire sa descendance agnatique, le lignage a alors recours au banni. L'union avec un banni permet d'assurer l'indivision du patrimoine lignager en évitant que la fille sans frère ou la veuve ne se marient avec un homme d'un autre lignage. Dès lors, le banni est soit capté par le lignage du père de sa femme, s'il s'agit d'une fille sans frère, soit par celui du beau-père de sa femme, si celle-ci est veuve.

La motivation du père qui n'a que des filles à accueillir un banni est de pouvoir garantir l'entretien et l'occupation de son patrimoine jusqu'à ce que celui-ci puisse être transmis à ses petits-fils, qui seront automatiquement intégrés dans sa filiation agnatique. Le banni est en effet appréhendé par les membres du lignage de sa femme comme un géniteur qu'ils cherchent à utiliser pour servir de relais dans la gestion des terres et de la résidence, le temps que ses fils, transformés en descendants "agnatiques" de leur grand-père maternel, en héritent la propriété. La stratégie est plus ou moins la même dans le cas de la veuve.

Le modèle du banni comme simple géniteur s'avère être, dans les faits, un idéal pour la famille d'accueil, idéal souvent contrecarré par l'ambition du banni lui-même à entrer dans l'histoire généalogique du lignage. Raymond Jamous, dans son étude sur les structures sociales dans le Rif, distingue deux modèles possibles d'intégration d'étrangers dans un lignage : soit l'étranger endosse le rôle du simple géniteur dont le nom sera effacé de la généalogie, soit « le nom de l'étranger n'est pas effacé, mais au contraire intégré dans le groupe agnatique : il devient le fondateur d'une lignée et même d'un segment de patrilignage » (Jamous 1981 : 52). Cependant, l'auteur ne s'attarde pas sur les conditions de l'effectivité de l'un ou l'autre de ces modèles. Pourquoi dans tel exemple, l'étranger joue-t-il le rôle d'un simple géniteur, alors que dans tel autre, il devient l'ancêtre fondateur d'un sous-lignage du groupe qui l'a accueilli ? 
Afin d'être reconnu comme un homme et un chef de famille, le banni n'a qu'une idée en tête, celle d'acquérir une propriété terrienne et résidentielle. Etant rattaché physiquement et matériellement à son lignage d'accueil, le seul moyen qui puisse lui permettre de devenir propriétaire est d'intercepter l'héritage qui transite entre les mains de sa femme. Si le banni la persuade de lui céder ses parts d'héritage de son vivant, il récupère une partie des biens immobiliers du lignage d'accueil en tant qu'héritier de la propriété de son beau-père (du père de sa femme) ou de l'ex-beau-père de sa femme (le père du premier mari défunt). Grâce au titre que le banni parvenu a obtenu de sa femme, son nom est affilié à une maison et à une portion de terre du patrimoine du lignage d'accueil. Il devient alors, vis-à-vis de ses membres, à la fois co-résident et co-propriétaire des terres.

Parmi toutes les catégories sociales, les chorfa (sing. chérif, descendant du prophète) sont ceux qui accueillent le plus de bannis. La raison en est que les chorfa sont dans l'obligation de préserver constamment l'indivision des territoires qu'ils occupent, garantie de la crédibilité de leur lien généalogique au saint fondateur de leur lignée et de leur statut de descendants du prophète Mohammed. Le banni est essentiel pour les chorfa car il permet de préserver l'indivision dans la consanguinité et, par là même, le lien généalogique aux saints fondateurs dont ils occupent les terres. Chez les chorfa, à partir du moment où le banni devient propriétaire des terres de sa femme, sa position dans le parcellaire lignager lui doit d'être assimilé à un consanguin, et cela afin qu'il ne titre pas individuellement sa parcelle, mettant ainsi en péril l'indivision et donc la filiation généalogique du groupe d'accueil. Son inscription dans le sol induit son inscription dans le sang. Qui plus est, l'acquisition du titre de chérif par les bannis semble bien participer au système de reproduction du chérifisme (système social fondé sur le rôle des descendants du prophète Mohammed). En effet, dans bien des cas, la descendance du banni fondateur se trouve dans une position favorable pour prendre les rênes du groupe généalogique qui l'a accueilli, et même en devenir le fleuron et le plus fidèle garant de ses valeurs sociales. Or, parmi les bannis qui ont été intégrés dans les lignages chorfa et qui ont fini par fonder les sous lignages les plus prestigieux, on retrouve un certain nombre de Juifs.

\subsection{L'histoire de la grande Maison des Zabun}

La grande Maison lignagère des Zabun est constituée d'un hameau à trois paliers qui domine le fond d'une vallée couverte d'arganiers et de figuiers de barbarie. Elle est connue pour être l'un des espaces sanctuaires (agdal) les plus renommés du pays Ait Ba'amran. La vallée en question est intégrée au territoire d'un village habité exclusivement par des chorfa Ouazzaniin (originaires de Ouazzane, au nord du Maroc) et qui marque la frontière nord-ouest 
du pays Aït Ba'amran. Lahcan Zabun, âgé de 76 ans en 2004, est le propriétaire de la totalité de la vallée sanctuaire qu'il gère d'une main de fer, de manière indivise. A ce titre, il est reconnu comme étant un pur chérif Ouazzaniin doté d'une grande baraka. Lahcan Zabun n'hésite d'ailleurs pas à déclamer avec orgueil, à qui ne le connaît pas ou à quiconque lui manquerait de respect, sa généalogie à dix générations qui le rattachent au saint fondateur de la lignée. Cependant, derrière son dos, tout le monde s'accorde à dire qu'il est en réalité le petit-fils d'un banni juif connu sous le nom de Ali, jadis chassé d'une communauté hébraïque présentée comme étant d'origine portugaise, Ouled Jerar, située à une centaine de kilomètres plus au nord.

Légende ou réalité ? Le rapport des Aït Ba'amran au Portugal est structurant dans leur manière de penser l'histoire. Si la présence portugaise le long de la côte marocaine et saharienne de Mogador à Boujdour est certes avérée, notamment sur les sites des anciens comptoirs commerciaux, la tradition orale Aït Ba'amran considère que les premiers colonisateurs de la région étaient portugais avant d'être espagnols. Les « Portugais» (Brtgz) incarnent ainsi la figure d'une allochtonie qui fut combattue par les saints de l'Islam (Simenel 2010).

Quoiqu'il en soit, les membres du lignage Zabun paraissent gênés quand on leur parle de cette histoire. Pour contredire la rumeur de leur origine juive portugaise, ils affirment que leur grand-père Ali, soupçonné d'être un banni juif, était en fait le fils d'un chérif Ouazzaniin du douar, qui aurait pris pour épouse une femme musulmane originaire des environs de la communauté hébraïque. Les Zabun racontent que ce chérif mourut alors que sa femme était enceinte ; celle-ci décida alors de repartir chez elle pour enfanter Ali, qui ne revint dans son douar paternel qu'à l'âge de 17 ans. Mais ce récit ne trouve aucun écho en dehors des membres du sous-lignage Zabun. Les habitants de la région contredisent cette version en expliquant que si Ali était bien, dès sa naissance, orphelin de père, il aurait dû, comme le veut la coutume, porter le même prénom que lui, en l'occurrence Mohammed.

Les habitants de la région racontent, en effet, sous forme de commérages, qu'Ali Zabun était un banni juif qui, à son arrivée, voici près d'une centaine d'année, se convertit à l'Islam et se maria avec une femme du dernier lignage Ida u Simelel du douar, une lignée chérifienne originaire de la région de Tafraoute (environ $200 \mathrm{~km}$ au nord-est) qui était en train d'être supplantée par celle des Ouazzaniin. La femme avec qui se maria le banni, n'avait, dit-on, que des sœurs, hormis un frère cadet dont le prénom, 'Abd, laisse supposer qu'il s'agissait non d'un frère mais d'un esclave. Cette femme était aussi la veuve d'un chérif Ouazzaniin. Les habitants de la région expliquent que, parlant et écrivant l'arabe tout en maîtrisant la magie - connue localement pour être une spécialité des Juifs - Ali Zabun parvint rapidement à prendre les fonctions de savant et d'officiant religieux (fqih) et devint célèbre dans la région pour la 
confection de talismans avant tout destinés à une clientèle féminine. Ayant acquis le statut de fqih et, par extension, celui de notaire ( ' $a d u l$ ) et de magicien, Ali Zabun n'eût aucun problème à persuader sa femme de lui léguer ses terres. Plus encore, grâce à la complicité intime qu'il avait tissée avec certaines sœurs de sa femme, restées célibataires et qui étaient aussi ses clientes, il réussit à devenir, en l'absence de parents masculins (hormis l'esclave élevé au rang de frère cadet), le responsable (amazel) de leurs terres. Petit à petit, il s'accapara ainsi la totalité des terres de la vallée qui revenaient aux chorfa Ida u Simelel, terres qu'il transmit à son fils. Parallèlement, le banni acquit au cours de sa vie un nom particulier, celui de Zabun. Comme ne cessent de le raconter avec passion les femmes chorfa de la région, Ali eût maintes fois l'occasion de se rendre dans les différentes tribus de la confédération pour prêcher. De la plus australe d'entre elles, il rapporta une nouvelle variété inerme de figuiers de barbarie, appelée « moïse » (moussa), qu'il réussit à implanter dans la vallée. Avec l'introduction de la variété «moïse » dans la région - introduction qui fut loin d'être négligeable du point de vue de l'apport alimentaire et économique - Ali acquit une certaine notoriété vis-à-vis des habitants qui le surnommèrent du nom arabe Zabun, la «figue de barbarie ». Ce surnom devint le patronyme de son sous-lignage.

A cette époque, la vallée était la propriété partagée des deux lignées chérifiennes, une partie revenant aux Ida u Simelel que le banni Ali Zabun s'appropria, une autre revenant aux Ouazzaniin. Ces derniers, qui occupaient l'autre moitié de la vallée, étaient alors conduits par un personnage religieux décrit, tant dans le discours oral des habitants de la région que dans les écrits rédigés par des historiens locaux tel Mokhtar Soussi, comme un grand savant mystique. Ce personnage charismatique, qui n'avait pour seule descendance que deux filles, est aussi présenté comme étant alors l'un des derniers descendants masculins de la lignée chérifienne des Ouazzaniin, installée dans la région. Mais sa ruse et sa perspicacité légendaires firent que le destin de sa lignée se joua habilement du sort qui s'acharnait à ne lui donner que des filles. En effet, à la mort du banni Ali, son jeune fils fut pris en charge par le grand savant qui devint pour lui un véritable tuteur doublé d'un maître spirituel. Le savant avait comme ambition de réunir l'ensemble de la vallée frontalière sous le sceau des chorfa Ouazzaniin. Pour arriver à ses fins, il devait donc au préalable intégrer le fils du banni dans sa lignée, puisque ce dernier avait hérité par son père de la partie de la vallée appartenant à l'autre lignée chérifienne. Pour ce faire, le savant maria le fils du banni avec une de ses petites cousines ; quant au père de ce dernier, il ne pouvait être effacé de l'histoire du lignage puisqu'il avait lui-même transmis la partie de la vallée qui revenait aux chorfa Ida u Simelel à son fils. Ali, le banni juif, fut alors positionné dans la descendance de l'oncle paternel du savant, en tant que frère du premier mari 
défunt de sa femme. Ce faisant, au nom d'une affiliation spirituelle et par le biais d'un mariage, le savant put dévier la filiation agnatique du sous-lignage Zabun vers sa lignée, et non plus vers la lignée des Ida u Simelel à laquelle ce sous-lignage était destiné à être intégré (Figure 2). Par ce coup de maitre, le grand savant perpétua la lignée chérifienne des Ouazzaniin en ces terres au dépend de celle des Ida u Simelel.

Figure 2. Parenté du banni Ali Zabun

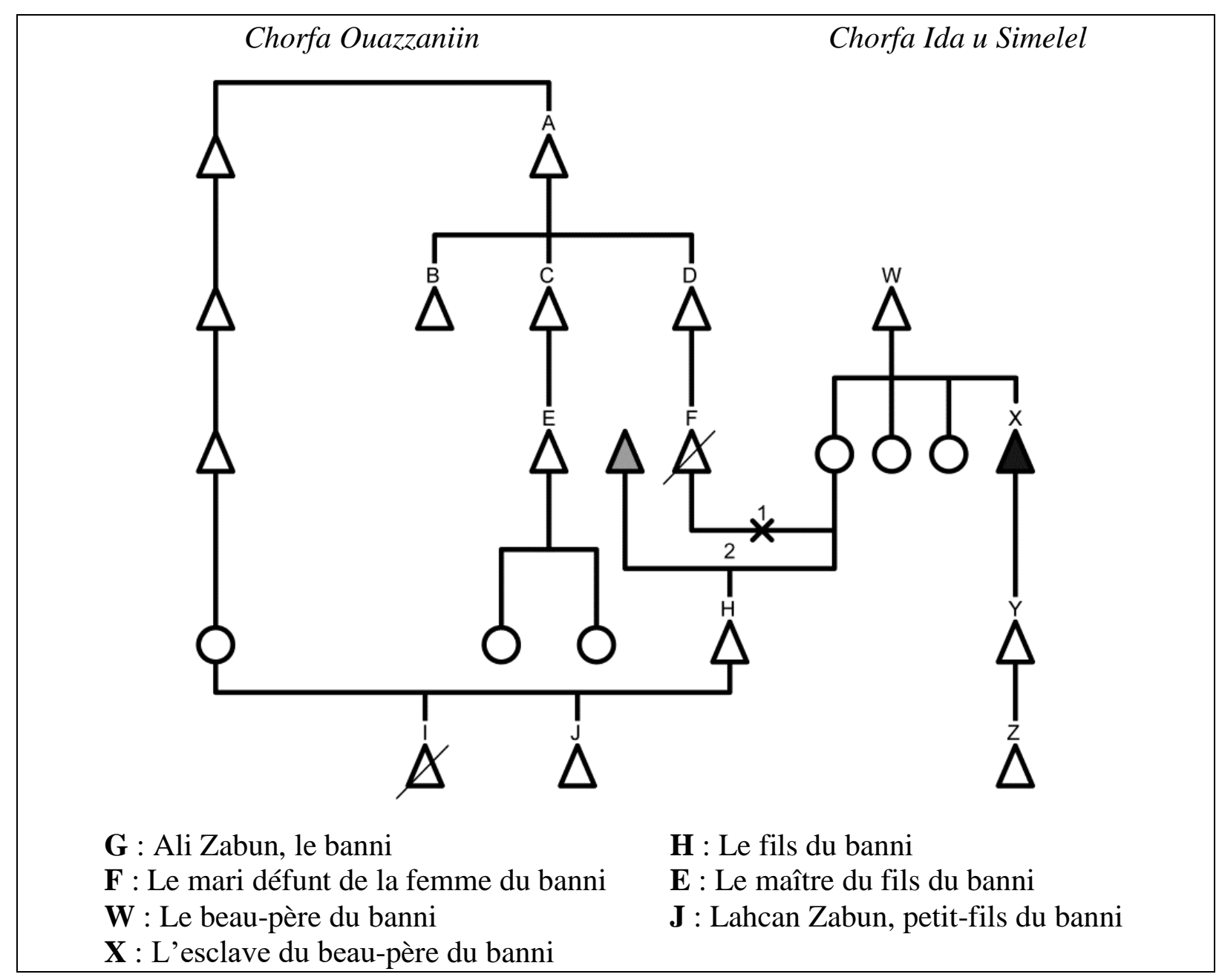

Source : Simenel, $2010: 286$.

Selon le discours oral, parmi les 59 autres étudiants du maître, figurent de nombreux ancêtres des lignages de chorfa Ouazzaniin habitant aujourd'hui la région. A l'image du fils du banni Zabun, les étudiants du grand savant étaient des étrangers qui ont pu intégrer la célèbre lignée chérifienne en partie grâce à l'entremise de leur maître spirituel. Ce dernier a donc joué le rôle d'entremetteur dans l'adoption d'étrangers au sein de sa lignée qui était menacée d'extinction. Pour ce qui est du fils du banni, positionné dans la généalogie du lignage de son maître spirituel comme son neveu en lignée agnatique, il a pu hériter des terres de l'autre partie 
de la vallée et c'est ainsi qu'il devint propriétaire de l'ensemble de la vallée en tant que chérif Ouazzaniin (Figure 3). Avant de mourir, on raconte que le maître avait minutieusement délimité la vallée frontalière pour en faire un espace sanctuaire hermétiquement fermé, un espace sanctuaire à la mesure de son savoir et de la destinée de son étudiant préféré, ce fils d'un banni juif qui s'affirma peu à peu, comme l'indique l'évolution de sa signature au cours de sa vie, comme un chérif Ouazzaniin, et donc un descendant du prophète Mohammed ${ }^{4}$.

Figure 3. La vallée des Zabun, «Aguni Zabun»

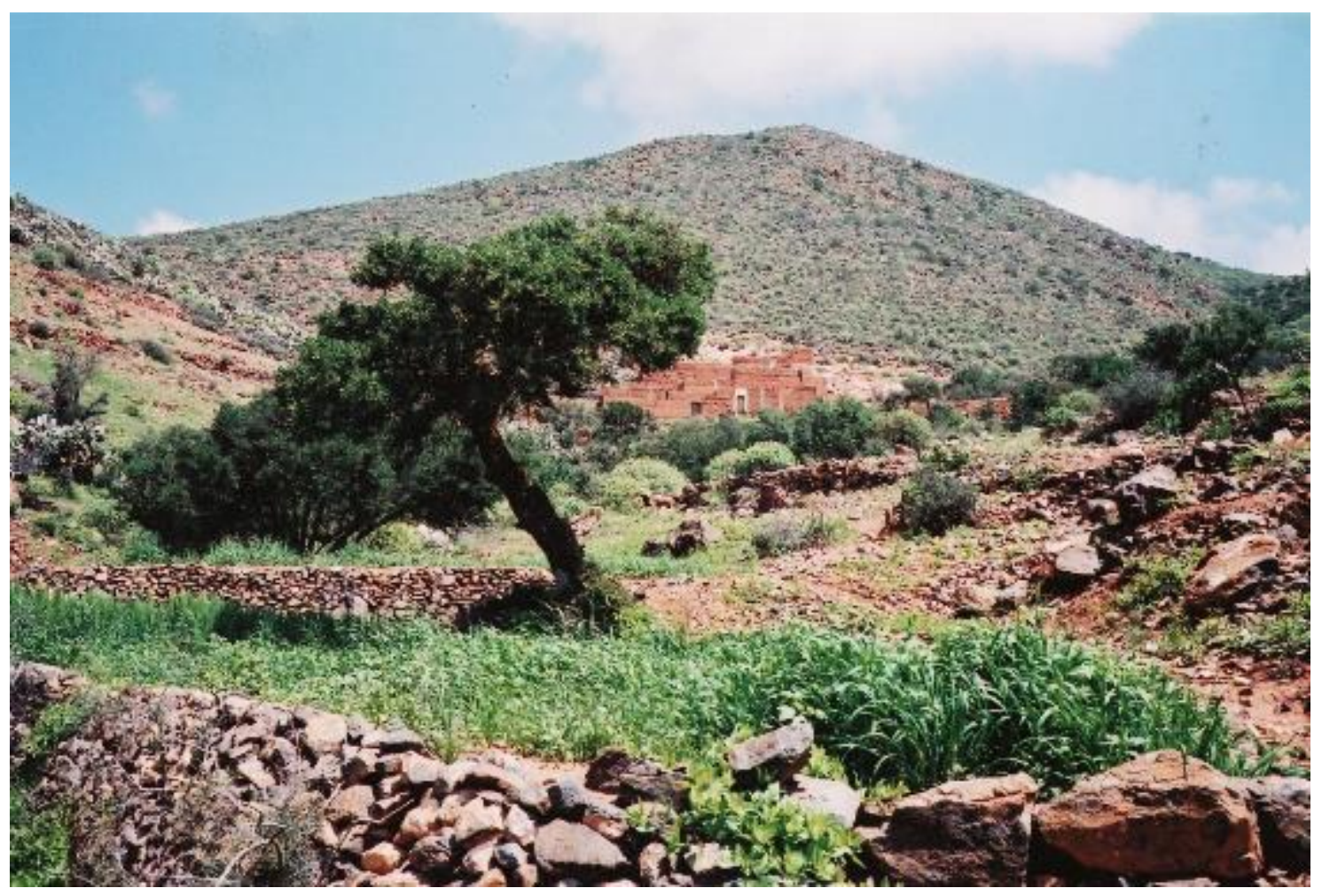

Photo : Romain Simenel, 2002.

De manière différente et complémentaire au récit biographique, le schéma de parenté et d'héritage ainsi que le schéma généalogique présentés ci-dessous (Figure 4) rendent compte du processus de constitution d'un sous-lignage chérifien à partir de la venue du banni juif. Le schéma de parenté et d'héritage retrace le processus d'infiltration et d'émergence du souslignage Zabun au sein de la lignée chérifienne des Ida u Simelel, puis au sein de celle des Ouazzaniin, tel qu'il est relaté par la rumeur. Le schéma généalogique restitue leur filiation agnatique chérifienne officielle, mise par écrit et reconnue de tous.

\footnotetext{
${ }^{4}$ Constatable à partir de la comparaison entre un acte d'antichrèse (1941) et une généalogie du saint Sidi M'hend ou Yussuf (1959).
} 
Figure 4. Parenté, héritage et généalogie du sous-lignage Zabun (G-H -J)

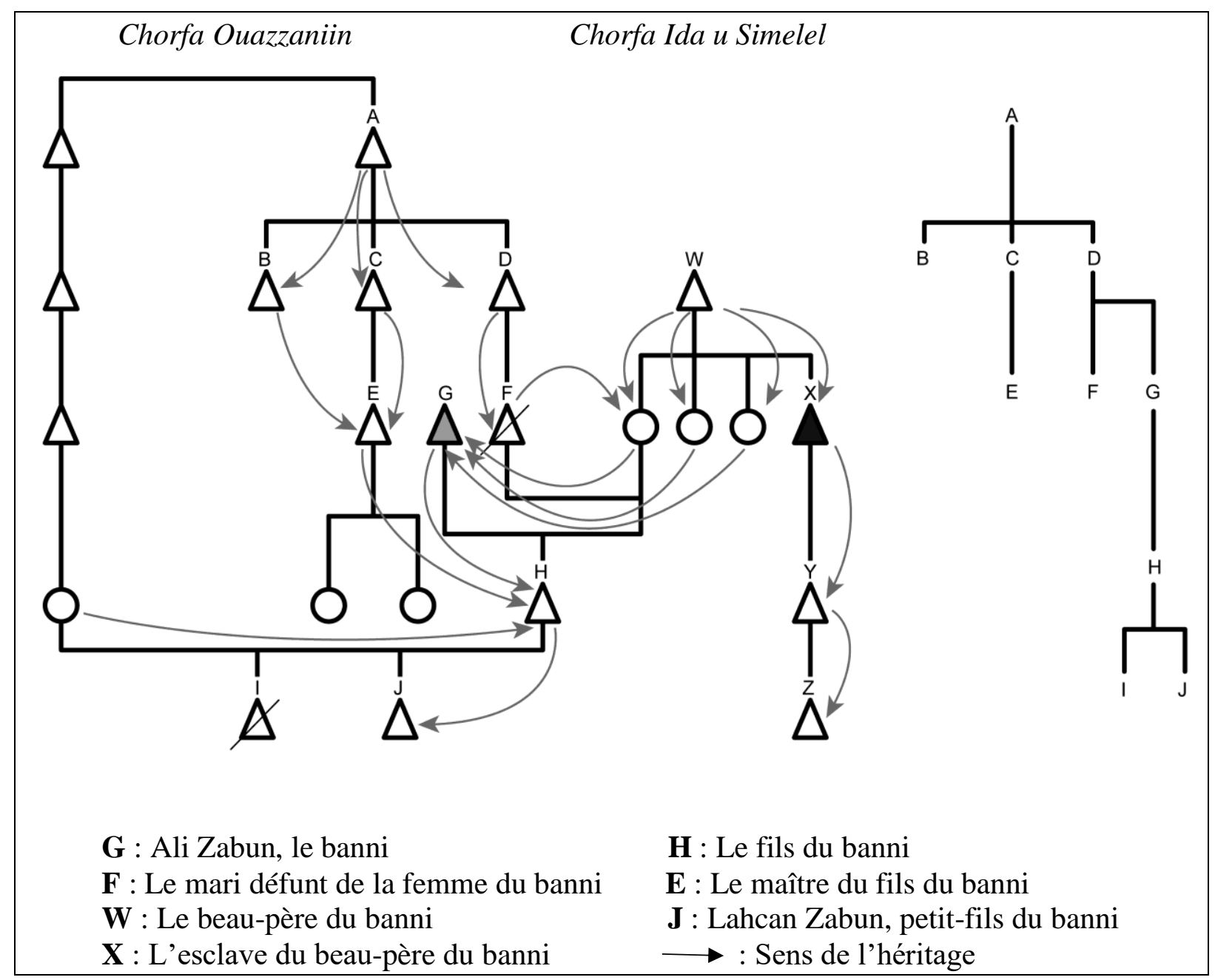

Source : Simenel $2010: 290$

\subsection{Pourquoi l'origine juive est-elle bonne à penser pour les lignages chérifiens ?}

Reste à répondre à la question suivante: pourquoi l'origine juive est-elle plus particulièrement présente dans l'histoire des lignages de chorfa ? Et, de manière corrélative, pourquoi, parmi tous les bannis, les Juifs sont-ils ceux privilégiés par les chorfa ? À la lumière de l'exemple de la grande Maison des Zabun, relaté par le discours oral, l'émergence du banni juif en tant que fondateur d'un sous-lignage au sein de son groupe d'accueil apparaît découler de sa capacité à séduire les femmes. Chez les chorfa, pour arriver à fonder son propre souslignage et à capter le statut de sa femme, le banni doit devenir au préalable le propriétaire des terres de cette dernière. Or, il ne peut y parvenir sans son aval. Chez les chorfa, ce sont les femmes sans frère et les veuves qui décident de l'avènement de nouveaux sous-lignages en choisissant de léguer ou non leurs terres à leur mari. Comme l'illustrent l'exemple de la grande Maison des Zabun et d'autres exemples fournis par le discours oral, le banni et ses fils jouent de leur caractère allogène pour séduire et s'attirer les faveurs des femmes de leur groupe 
d'accueil (Simenel 2010). La maîtrise de l'écriture de l'arabe et les connaissances religieuses, magiques ou poétiques d'un banni sont considérées comme autant d'atouts de son origine étrangère. Le banni et ses proches descendants endossent un statut d'officiant religieux ( $f q i h)$, et ce, du fait qu'ils viennent de loin, c'est-à-dire de régions qui, en vertu de leur éloignement, sont d'emblée considérées comme plus érudites. Le rôle de magicien tenu auprès des femmes par un banni ayant endossé le statut de fqih est certainement l'une des voies les plus courantes de son insertion dans l'histoire des lignages. Les femmes sont avides de nouveaux talismans ou de recettes magiques venant de régions réputées savantes, et un banni qui possède quelques notions de magie peut s'en servir pour les séduire. Un banni revêtant le rôle de magicien, est à même de tisser une complicité avec les femmes sans égal dans la société, et exerce une influence directe sur la reproduction du groupe. Or, les Juifs étaient justement réputés pour détenir la magie la plus redoutable. La préférence des lignages chorfa pour les bannis juifs reposerait ainsi en partie sur l'attrait des femmes pour leurs savoirs magiques.

Figure 5. La fille et le petit fils de Lahcan Zabun

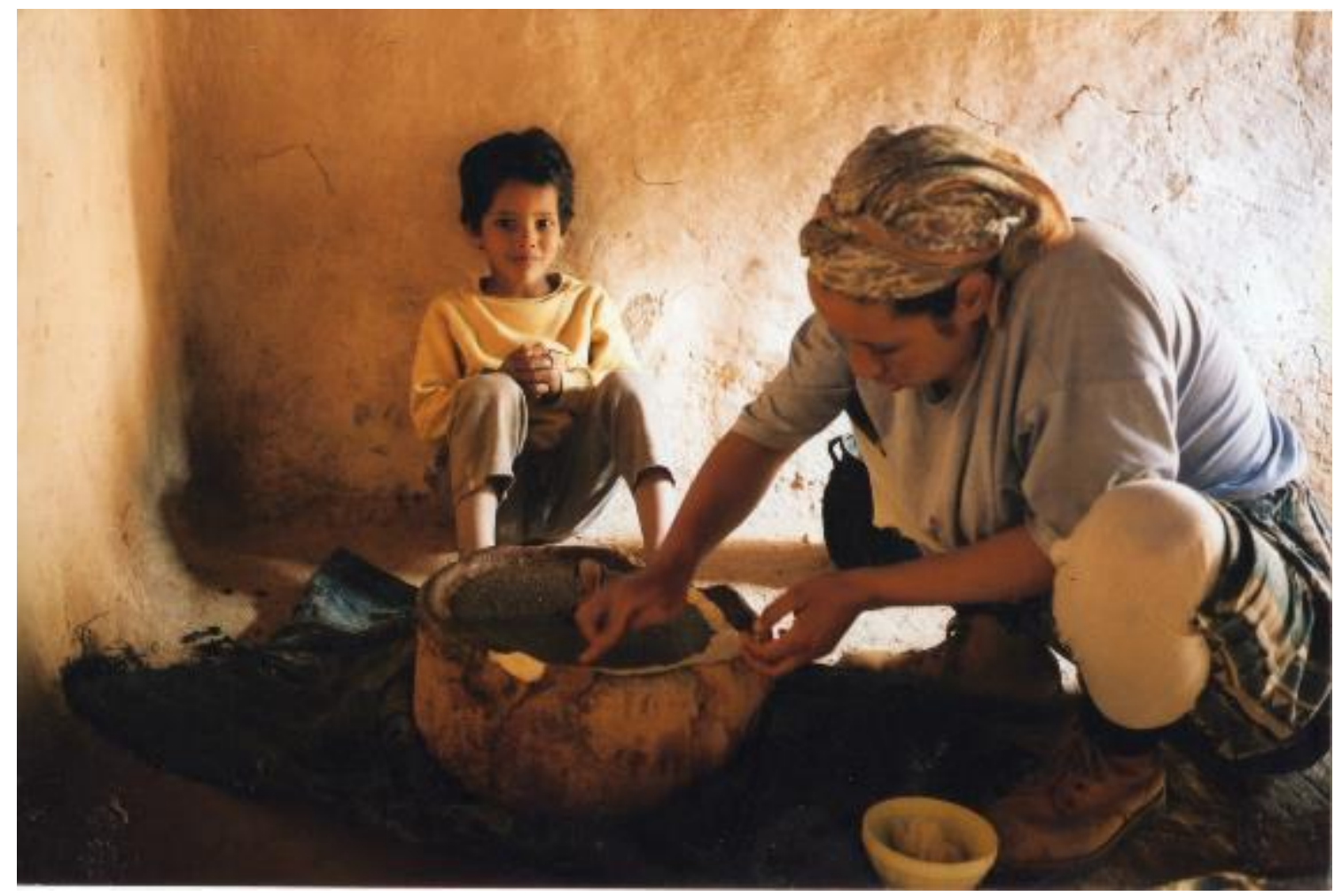

Photo : Romain Simenel, 2002.

Le plus souvent, la descendance masculine du banni hérite de sa compétence et en use pour séduire plusieurs femmes afin de constituer un foyer polygame. Comme les femmes aiment à le raconter, le banni et sa descendance constituent aussi une filière par laquelle la 
société s'ouvre à de nouveaux produits, comme par exemple une variété originale de figuier de barbarie ou de plante médicinale, à de nouvelles techniques, comme le greffage arboricole, ou encore à de nouveaux matériaux comme le ciment. Le banni parvenu et ses fils restent dans l'histoire comme ceux qui ont apporté une "touche personnelle" au terroir de leur lignage d'accueil, à l'instar d'Ali Zabun avec la figue de barbarie sans épines. Les femmes se souviennent ainsi du banni parvenu comme quelqu'un qui a perfectionné la culture spirituelle et matérielle locale, qui l'a enrichie, et c'est en tant que tel qu'elles le présentent comme un fondateur de lignage. Non seulement le banni renouvelle la parenté du groupe d'accueil, mais en plus, il reste dans l'histoire comme celui qui a rénové la culture locale. Et même si la confession juive du banni est niée par ses descendants, elle reste actualisée par la rumeur et par tous ces indices tangibles comme les patronymes ou toponymes afin de ne jamais oublier que la transmission des origines ne peut se reproduire que par l'ailleurs.

Terre d'accueil, le Maroc fut aussi une terre de départ pour les Juifs vers d'autres horizons. C'est ainsi que certains d'entre eux, partirent en Amazonie pour participer à une autre histoire généalogique.

\section{L'origine juive dans la construction du lien au sol en Amazonie}

\subsection{Des « Juifs » dans la région du bas-Amazone brésilien}

L'ethnologue à l'écoute des récits des populations riveraines de la région du basAmazone - qu'elles soient urbaines ou rurales, issues de milieux sociaux variés et identifiées sous des catégories identitaires distinctes - est surpris par l'omniprésence des discours sur les origines «juives » de ses interlocuteurs. Presque systématiquement, le narrateur se présente comme étant le résultat d'un « grand mélange » (é uma grande mistura) composé d'immigrés «blancs » dont l'origine géographique est connue précisément (le Portugal, l'Italie, etc.), $\mathrm{d}^{\prime}$ «Indiens » (índios) $)^{5}$ et/ou d'esclaves africains. Cette allochtonie généalogique est parfois présentée comme un cumul ; par exemple, un immigré de la région Nord-est du Brésil et d'origine portugaise, ou un juif du Maroc et d'origine andalouse. Pour nos interlocuteurs, le métissage est souvent constitutif de l'autochtonie amazonienne. Le mélange des origines et la figure de l'étranger intégré (introsado) proportionnent l'ancrage culturel et territorial des populations. Ainsi, les personnes se présentent comme descendant d'individus venus d'ailleurs, d'un autre village, d'une autre rivière, d'une autre région ou d'un autre pays.

\footnotetext{
${ }^{5}$ Terme dépréciatif qui évoque la figure du sauvage et les stéréotypes associés (anthropophage, nu, incestueux). Sur le contraste entre « indígena civilizado » et « índio brabo », voir Boyer (2015: 14) et Stoll (2014).
} 


\section{Récits de fuites et dissimulation des patronymes juifs}

Dans ce tableau général amazonien, l'immigré juif est souvent cité. Pour les populations de la région de Santarém, la judaïté est davantage une origine (géographique, voire phénotypique) qu'une adhésion religieuse (Nugent 2009 : 108). Dans la plupart des cas, elle est marocaine. Mais la figure de l'immigré juif inclut aussi les descendants de nouveaux-chrétiens et des populations de confession hébraïque arrivées plus tardivement.

La judaïté des origines est décrite avec des motifs récurrents qui font écho à ceux de l'imaginaire juif globalisé. Le premier est celui de la fuite : «ils sont arrivés ici en se sauvant » (chegaram fugidos). Le lieu d'origine du fuyard reste assez flou. L'interlocuteur hésite avant de s'exclamer : «il venait ... de Judée ! c'est un juif, il s'est enfui de Judée » (da Judeia, chegaram fugidos da Judeia). L'arrivée se fait par voie fluviale : «ils sont arrivés par le fleuve » (boiando por aí) ou encore « dans une caravelle ». Le deuxième motif invoqué est celui $\mathrm{du}$ « faux nom ». Le fugitif a changé de patronyme afin de camoufler son origine juive, dans sa fuite ou pour les besoins de la naturalisation. On entend fréquemment dire que les familles possédant un nom d'objet, de plante ou d'animal seraient d'origine juive. Cette idée exprimée par les petits agriculteurs familiaux d'Amazonie est un lieu-commun de l'univers marrane pour évoquer l'histoire des convertis - les nouveaux-chrétiens (Benchimol 2008 : 197). À Santarém, sont ainsi identifiées les familles Machado (hache), Pinto (poussin), Mattos (forêt), Oliveira (olivier), Figueira (figuier), Pereira (poirier), etc. Il s'agit pour la plupart des patronymes des familles de notables, installées à Santarém dès le XVIIe siècle. Ainsi certains descendants du Baron du Tapajós (famille Corrêa, du mot « courroie »), revendiquent une origine juive. Cette famille aisée, «blanche » selon les critères locaux, se présente aussi d'origine portugaise. Ces données évoquent un phénomène historique bien étudié par Nathan Wachtel $(2011,2001)$ : la diaspora des nouveaux-chrétiens vers les Amériques. D'après Wachtel, «sur le continent américain, c'est au Brésil que les nouveaux-chrétiens portugais émigrèrent en plus grand nombre et y constituèrent [...] la population la plus dense », formant, dès le début de la colonisation, «la majorité de la population blanche», dans une proportion estimée à un portugais sur trois $(2011: 12,13)$.

\section{L'arrivée des Juifs séfarades en Amazonie et le développement du commerce}

La présence juive en Amazonie dépasse les descendants de nouveaux-chrétiens. Dès 1810, des réseaux migratoires sépharades se mettent en place, la plupart en provenance du Maroc. Cette période coïncide avec une intensification du commerce international suite à 
1'ouverture de l'Amazone à la navigation internationale ${ }^{6}$, et à un accroissement des libertés individuelles et religieuses. La plupart des immigrés juifs vient alors du Maroc - principalement de Tanger, Tétouan, Fez, Rabat, Salé, Marrakech (Benchimol 2008 : 15). Ces immigrés sont pour la plupart d'origine portugaise et espagnole. Arrivés en Amazonie par Belém, ville portuaire située à l'embouchure de l'Amazone, ils remontent le fleuve et s'installent dans les villes riveraines de l'arrière-pays, dont Santarém, lieu de cette étude. D'après les recensements des cimetières israélites, environ 300 familles juives sépharades s'installent en Amazonie entre 1810 et 1850 ; puis 700 autres pendant la période du cycle du caoutchouc, entre 1851 et 1910 (Benchimol 2008 : 74). Trois autres vagues de Juifs arrivent au XIXe siècle : une centaine de familles juives françaises fuient l'Alsace-Lorraine pendant la guerre franco-prussienne (18701871) et s'installent dans cette région (limitrophe de la Guyane) où ils investissent dans le commerce du latex. Une autre centaine de familles juives ashkénazes originaires d'Allemagne et de Pologne s'établissent comme commerçants, propriétaires de boutiques et exportateurs de latex (Benchimol 2008 : 77). Enfin, un autre contingent d'immigrés juifs, très présent dans l'imaginaire amazonien, les «Turcs» (Turcos), arrive de Turquie, du Liban, de Syrie et d'Égypte, et occupe des emplois de commerçants navigants et de vendeurs ambulants en ville.

À la fin du $\mathrm{XIX}^{\mathrm{e}}$ et au début du XX $\mathrm{X}^{\mathrm{e}}$ siècles, la population juive d'Amazonie est très active dans les réseaux commerciaux, à différentes échelles (du vendeur ambulant au propriétaire de magasin en passant par les commerçants établis en zone rurale). Elle travaille en étroite collaboration avec d'autres collectifs de migrants auxquels ils sont parfois assimilés, comme les Français ou les Espagnols. En 1886, l'explorateur français Henri Coudreau estime qu'environ 200 juifs francophones commercent le long du bas-Amazone (Coudreau 1886 : 365). À part à Belém et Manaus où ont été construites des synagogues, la religion juive ne s'est pas maintenue dans les petites villes amazoniennes. Même si la plupart possède des cimetières israélites, on observe plutôt une tendance de ces collectifs à s'intégrer dans la population régionale par des unions exogames. Il ressort de nos enquêtes que l'origine juive tend à devenir un lieu exogène davantage qu'une confession religieuse dans un mouvement plus large de constitution des sociétés amazoniennes. Explorons désormais ce processus d'ancrage à partir de la trajectoire d'une famille, celle du juif Lévi.

\footnotetext{
${ }^{6}$ Charte royale du 28 janvier 1808 sur l'Ouverture des ports luso-brésiliens aux Nations amies et son décret d'application du 18 juin 1814 .
} 


\subsection{L’ancrage territorial du juif Lévi par la nomination et la généalogie}

La trajectoire du juif Lévi et de ses descendants - la famille Tapajós - le long des rivières Jary et Arapiuns, dans la commune de Santarém, est un exemple paradigmatique de la façon dont une famille d'immigrés s'intègre dans le paysage social et géographique amazonien et s'autochtonise tout en conservant la mémoire de son origine exogène singulière.

\section{Un juif « sans nom » baptisé Tapajós}

Comme dans la plupart des récits sur les origines juives, l'histoire du juif Lévi débute par un dilemme de patronyme. Selon ses nombreux descendants, Lévi s’était « enfui de Judée » et, parce qu'il était juif, «il ne possédait pas de nom de famille». En arrivant dans la région de Santarém vers 1820, Lévi se fit «baptiser » et reçut un nom brésilien : Luiz Caetano Tapajós. C'est sous ce nouveau nom - un « faux nom, pour se cacher »-que Lévi alias Luiz Caetano est remémoré par ses descendants. C'est également sous ce nom qu'il signera, en 1891, son testament. On y apprend que, fils de Sine et Samuel Cohen, il est né à Mogador, au Maroc. Luiz Caetano Tapajós est un juif sépharade venu tenter sa chance en Amazonie.

Notons que le patronyme choisi - Tapajós - est le nom de la rivière au bord de laquelle est érigé Boim, la localité où il est baptisé. C'est également en aval de cette même rivière que Luiz Caetano s'installera comme commerçant et cultivateur de cacao (Figure 6). L'acte de nomination ancre immédiatement l'immigré dans la toponymie et la topographie locales. Cette action, réalisée à l'église sous la forme d'un baptême, efface la confession hébraïque de son porteur. Une fois acquis ce patronyme et la religion catholique, Luiz Caetano peut à son tour le transmettre, et ainsi nommer ses enfants et d'éventuels filleuls. Le nouveau nom autorise la fondation d'une nouvelle lignée ancrée spatialement et symboliquement dans le bas-Amazone. 
Figure 6. Carte de la région du bas-Tapajós, commune de Santarém - Pará

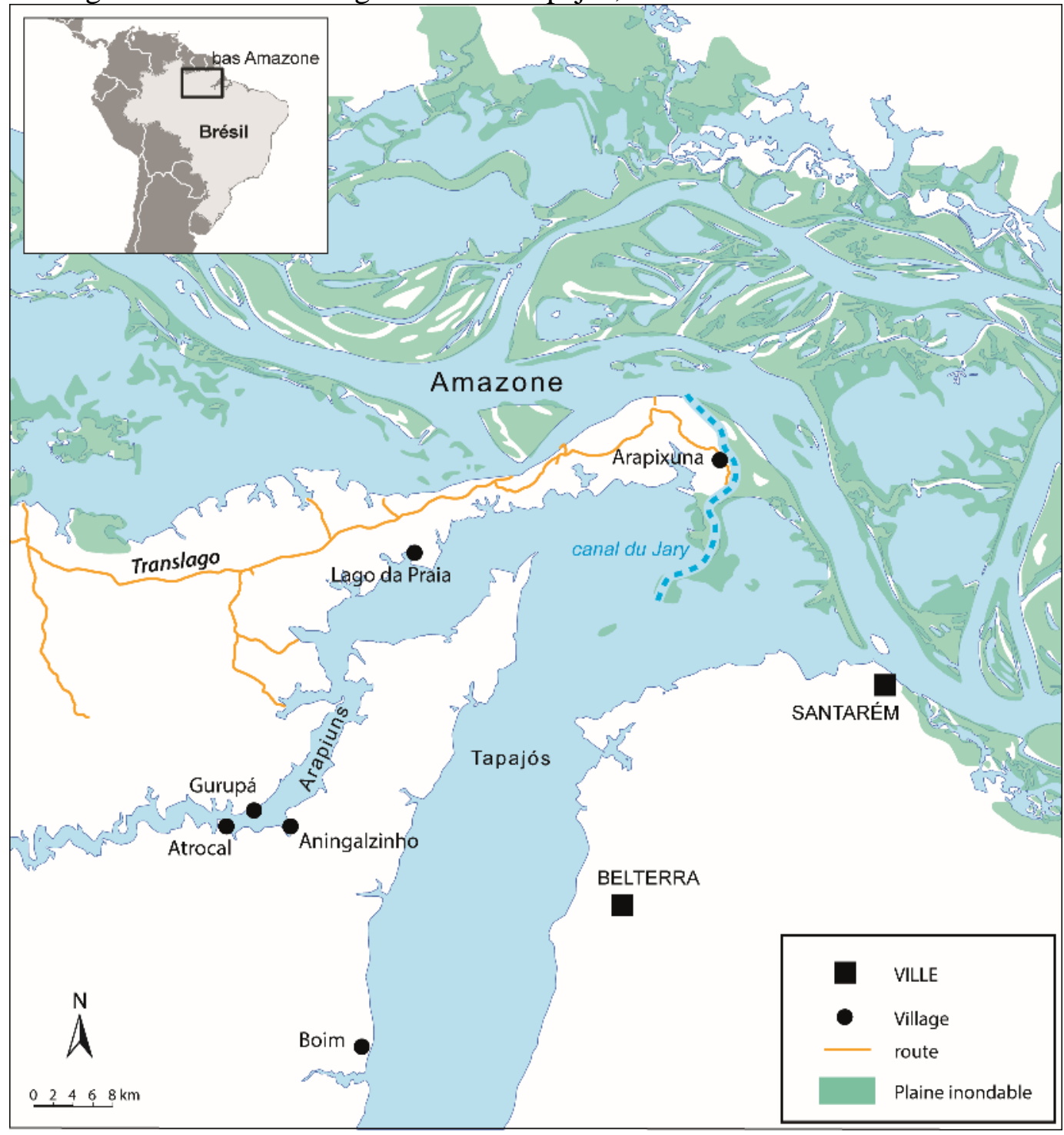

Réalisation : Laurence Billault - IRD.

\section{Un immigré commerçant fondateur du lieu Jary}

Dans la plaine d'inondation (écosystème fertile inondé par la crue annuelle), Luiz Caetano Tapajós s'installe au bord du Jary, un canal qui traverse la péninsule Arapixuna du Sud au Nord, reliant la rivière Tapajós au fleuve Amazone (Figure 6). Cette région est un haut lieu de culture du cacao jusqu'au début du XXe siècle. Selon une des versions, « en 1825, à la recherche de terres vierges pour planter du cacao, et suite à l'ouverture des ports brésiliens au commerce international et à la forte demande pour ce produit, le juif Lévi remonta la rivière jusqu'au lieu qu'il nomma Capintuba, où il établit ses activités, et nomma le cours d'eau 'Juri' [origine de Jary] » (Miguel Pinto ${ }^{7}$ ). Pour ses descendants, Luiz Caetano Tapajós aurait ainsi

\footnotetext{
${ }^{7}$ Historien autodidacte descendant du juif Levi.
} 
nommé la rivière Jary, une version contestée par les autres familles qui s'y établirent avant lui ou à la même période.

D’après son inventaire successoral, Luiz Caetano Tapajós possédait un entrepôt commercial ainsi qu'une maison relativement luxueuse, puisqu'outre deux alcôves et une véranda (Figure 7), elle était couverte de tuiles et décorée de céramiques portugaises (azulejos), une vraie distinction sociale à cette époque. Aujourd'hui encore, il est possible d'apercevoir les vestiges du promontoire construit par les esclaves, au-dessus duquel était érigé l'entrepôt. Dans le canal du Jary, toutes les familles de planteurs de cacao se revendiquent d'ascendance étrangère et possédaient des esclaves.

Figure 7. Maison d'un arrière-petit-fils de Luiz Caetano Tapajós au bord du Jary L'élevage bovin a aujourd'hui remplacé les plantations de cacao

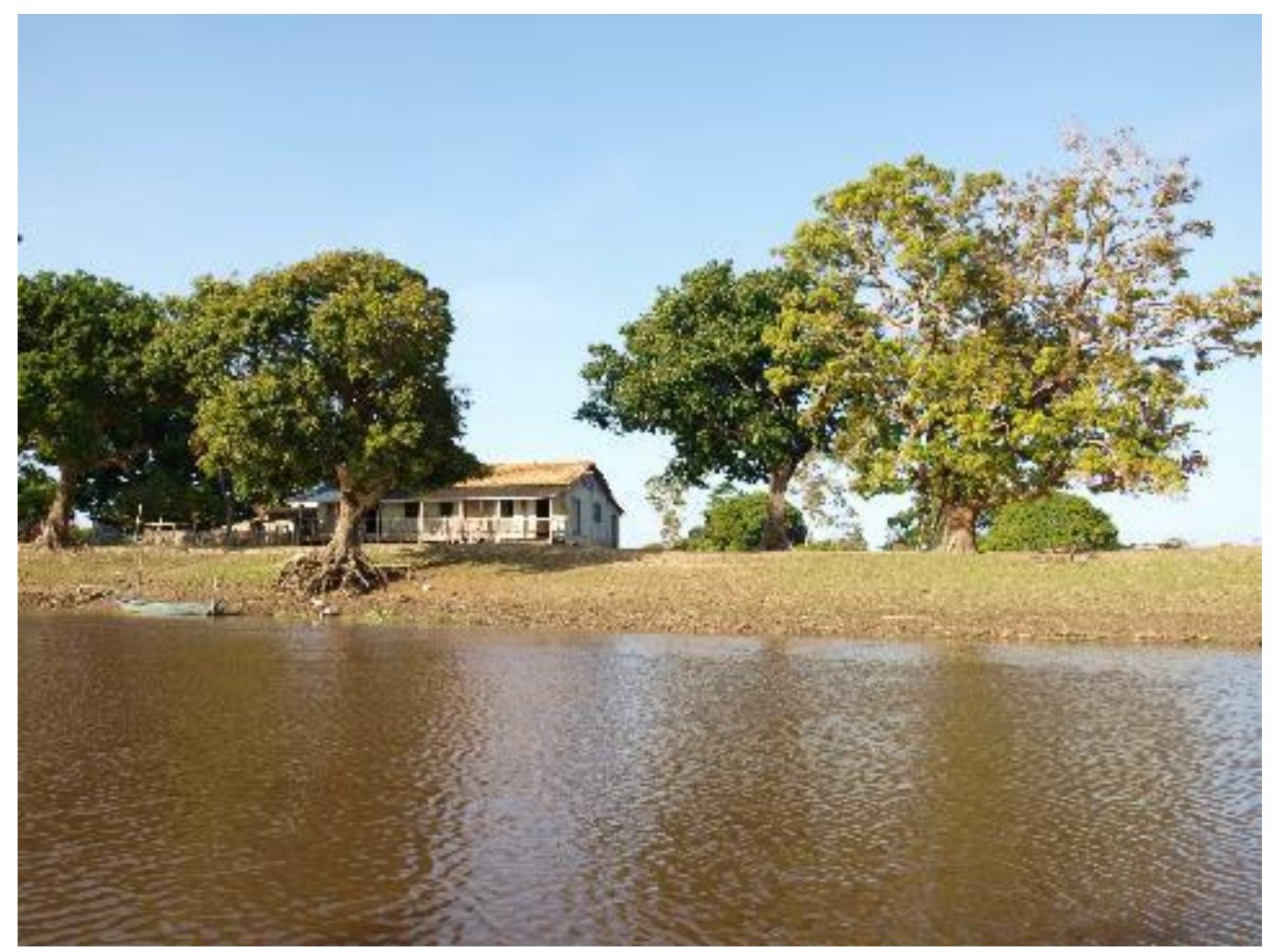

Photo : Emilie Stoll, 2018.

\section{La construction de la famille Tapajós}

En tant qu'étranger, à la sortie de la période coloniale (1822), Luiz Caetano Tapajós est le gendre idéal. Il ne possède pas de famille, mais est porteur d'autres savoirs - l'art du commerce - et de compétences singulières, notamment un capital génétique. Comme le répètent à loisir ses descendants, « il était blanc aux yeux bleus » (Figure 8). En effet, tout au long du $\mathrm{XIX}^{\mathrm{e}}$ siècle, au Brésil, va se construire un imaginaire national d'une population née de la fusion 
des «trois races » (Amérindien, Africains, Portugais), mais qu'il convient de « blanchir » grâce à un contingent d'immigrés blancs afin de contribuer à la marche vers le progrès (Rozeaux 2014), notamment les populations d'Amazonie, considérées arriérées et peu aptes au travail agricole. Paul Le Cointe, immigré français installé dans la région de Santarém, écrit à ce sujet : «Un ramassis de criminels et d'aventuriers sans foi ni loi a été la souche principale de l'élément blanc qui allait se développer à côté de l'Indien qu'il ne pouvait que pervertir, et c'est d'elle que descend la population métisse [d'Amazonie], aujourd'hui la plus importante, par l'union, tout d'abord presqu'exclusive, de pères européens et de mères indiennes. Elle devait se ressentir longtemps de ces origines, et il fallait l'infusion, sans cesse renouvelée, de sang plus pur, pour hâter le lent travail naturel de régénération » (Le Cointe 1922 : 218-220)

Entre 1850 et la première décennie du XXe siècle, le Brésil puis l'État du Pará vont mettre en place des politiques visant à faciliter l'établissement de colons étrangers (Stoll et al. 2017). C'est dans ce contexte socio-économique très favorable aux immigrés blancs que s'inscrit la trajectoire sociale et économique des juifs séfarades.

Figure 8. Un petit-fils de Luiz Caetano Tapajós et sa fille dans leur véranda

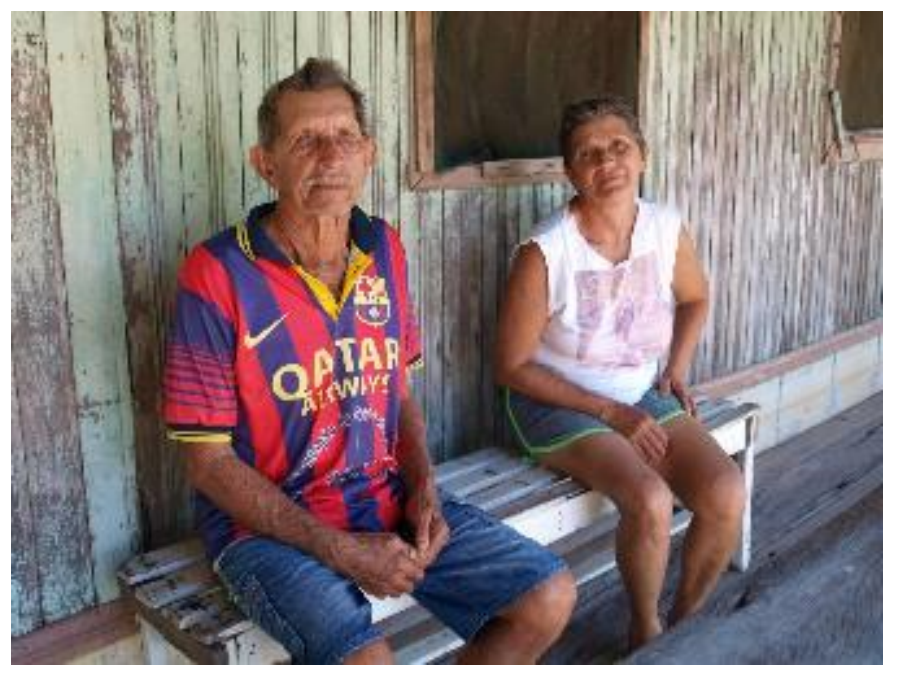

Photo : Emilie Stoll, 2018.

Luiz Caetano Tapajós s'est marié trois fois. Ses trois épouses - Joanna Nogueira, Angela Joaquina de Oliveira et Joanna Corrêa - sont issues de familles de notables de la région de Arapixuna. De ces trois unions, il eut 4 enfants, trois filles et un garçon. On sait, grâce à l'inventaire successoral et aux récits oraux des descendants, que la fille aînée - Virginia - était partie sans plus donner de nouvelles. La cadette - Maria - avait épousé un homme aisé peu 
attaché à la terre du Jary. Le couple partit s'installer en ville. Le troisième enfant Hidelbrandina - épousa le vacher noir de son beau-frère, contre l'assentiment parental. C'est sans doute pour ces raisons que Luiz Caetano Tapajós lègue la plus grosse partie de son héritage à son fils Jayme, à travers la réserve héréditaire. Ce sont donc les trois enfants de Jayme Antonio Messenas, Carolina et Luiz Tupaiu - qui vont perpétuer la lignée du Juif Lévi et se transmettre ses terres du Jary (Figure 9).

Figure 9. Généalogie du Juif Lévi et unions endogames de ses descendants

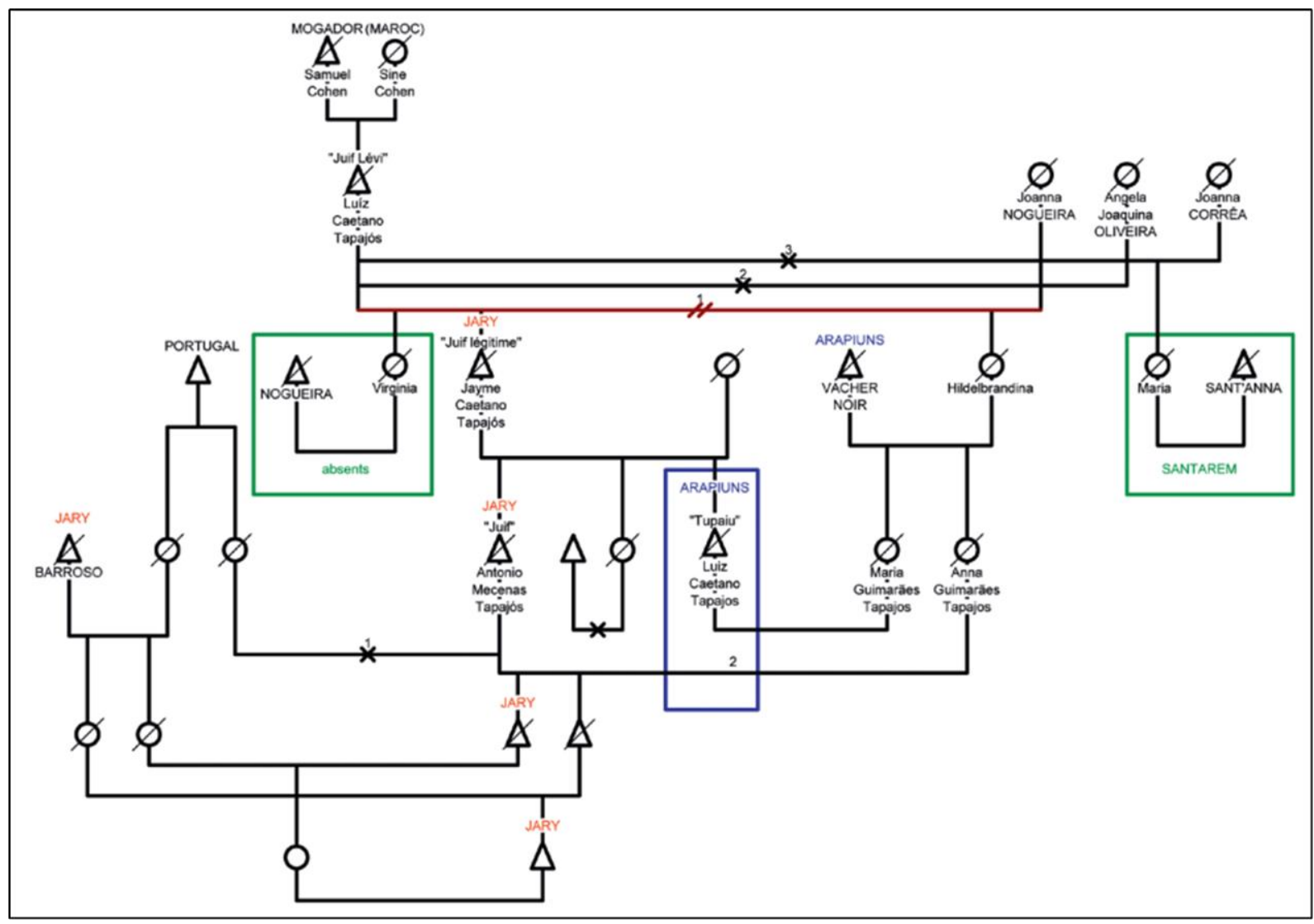

Sources : enquêtes de terrain, livres de baptême et de mariage du diocèse de Santarém, testament et inventaire successoral de Luiz Caetano Tapajós.

Or, cette famille va privilégier des unions endogames sur le modèle du mariage entre cousins croisés, notamment entre les familles de Jayme et de sa sœur Hidelbrandina, et les renforcer sur un modèle similaire avec deux autres familles propriétaires terriennes du Jary d'ascendance portugaise (Figure 9). Cet entre-soi matrimonial a pour objectif de conserver le capital génétique des aïeuls blancs (juifs et portugais), en particulier dans un espace où les familles descendantes de colons côtoyaient les descendants d'esclaves africains. Ainsi, « autrefois, les gens ne voulaient pas mélanger le sang avec les noirs » (não queriam espalhar o sangue com os pretos). Les unions mixtes étaient mal vues. Aujourd'hui encore, les habitants du Jary portent les traces physiques de ces unions endogames ; beaucoup ont les yeux bleus- 
verts et la peau plus claire que la moyenne régionale (Figure 8). Lorsque les unions échappent à cette règle - par exemple le mariage entre Hidelbrandina et le vacher noir - les stratégies de mariage endogamiques des enfants issus du couple tendent à « effacer » un apport génétique non désirable sur la génération suivante. Par ailleurs, ces stratégies matrimoniales permettent de conserver un patrimoine foncier relativement indivis (du point de vue du groupe résidentiel dans son ensemble) entre les mains des descendants restés dans le Jary, à savoir les enfants de Jayme et Hidelbrandina.

\subsection{De « juif légitime » à « Indigènes Tupaiu »}

Si le discours dominant au sein des parentèles Tapajós du Jary est celui de l'endogamie à visée phénotypique, ce n'est pas le cas au sein de celles établies le long de la rivière Arapiuns voisine. Ce constat est visible dans les trajectoires différenciées des deux fils de Jayme (fils héritier de Luiz Caetano Tapajós) - Antonio Massena et Luiz Tupaiu - et des choix matrimoniaux et identitaires de leur descendance. Aujourd'hui, les Tapajós du Jary s'identifient comme «grands blancs» (brancões) de la plaine d'inondation, et ceux de l'Arapiuns comme « Indigènes » de l'ethnie Tupaiu.

\section{Deux frères aux trajectoires divergentes : Antônio Tapajós et Luiz Tupaiu}

Antônio Massena Tapajós, fils aîné de Jayme, est remémoré par ses enfants et petitsenfants comme «un juif légitime ». Marié en premières noces à la fille d'un portugais, il épousera en secondes noces sa cousine Anna, fille métisse de sa tante Hidelbrandina. Anna Guimarães Tapajós est décrite comme une femme noire (uma morena), avec laquelle il aura de nombreux enfants. Antônio Massena, un homme « respecté », était propriétaire d'esclaves ; il hébergeait un vieux noir dans le fonds de sa maison bien après l'abolition de l'esclavage. Cette parentèle nombreuse a poursuivi des unions endogames et a prospéré économiquement, notamment grâce à l'élevage bovin, après la destruction des plantations de cacao par les grandes crues des années 1950.

Le frère cadet, Luiz Caetano Tapajós (petit-fils), a épousé la sœur de l'épouse de son frère, qui n'est autre que sa cousine croisée patrilinéaire Maria Guimarães. En plus d'être vacher, le père de Maria et de Anna Guimarães était originaire de la rivière Arapiuns où se déverse le canal du Jary. L'Arapiuns est une rivière peu poissonneuse située dans un écosystème moins fertile que la plaine d'inondation. Dans la région, elle est considérée comme une terre « de la faim », habitée par « des pauvres, des noirs et des índios ». Or, c'est au bord de cette rivière que Luiz s'installe avec sa famille. Dans le contexte social et géographique de 
l'Arapiuns, il devient Luiz « Tupaiu », un surnom peu flatteur qui évoque l'indianité et plus précisément un préjugé attribué aux « índios »: le mauvais payeur. Luiz est remémoré comme un habile constructeur de bateaux. Il entretient des liens d'amitié et d'échange commercial avec ses neveux du Jary, notamment les fils de son frère Antônio Massena, qui viennent dans les centres forestiers de l'Arapiuns acheter du bois et faire des affaires.

Le long de la rivière Arapiuns, les pratiques matrimoniales sont exogames : on se marie au plus loin généalogiquement et géographiquement (Stoll 2014). Les unions entre cousins germains sont mal perçues et on évite les mariages avec les voisins immédiats, source de disputes. L'objectif est davantage de créer des groupes résidentiels suffisamment nombreux pour assurer un réseau de coopération et une force de travail utiles à l'agriculture sur brûlis. Les descendants de Luiz Tupaiu se sont ainsi largement mélangés à la population régionale grâce à des unions exogames suivant d'autres logiques que celle de la sélection génétique. Ils ont un mode de vie différent de celui de leurs parents du Jary. Ils vivent de la production de farine de manioc et pratiquent l'agriculture vivrière. Ils résident aujourd'hui dans différents villages de l'Arapiuns. Contrairement aux descendants de Antônio Massena, ceux de Luiz Tupaiu ont connu une trajectoire socio-économique plutôt descendante, visible, par exemple, par leurs maisons en palmes (Figure 10).

Figure 10. Descendants de Luiz Caetano Tapajós devant leur maison dans un village indigène, Arapiuns
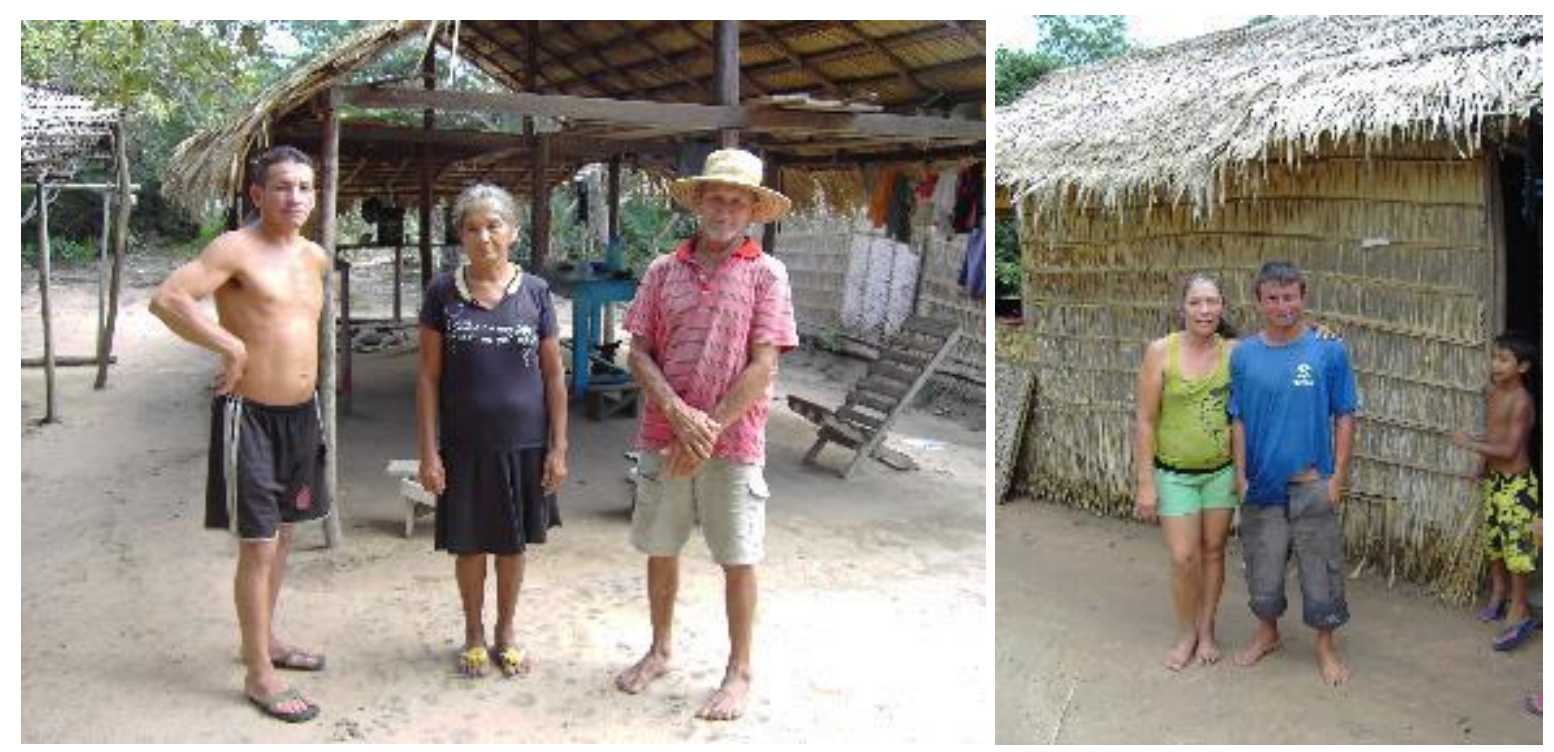

Photo : Emilie Stoll, 2011. 


\section{L'héritage juif dans l'identification à l'indianité}

Depuis les années 2000, les parentèles Tapajós installées le long de l'Arapiuns s'identifient comme indígenas ${ }^{8}$ et demandent la création de Terres Indigènes à l'État brésilien. Or, la mise en avant de cette identité légale ne signifie pas un camouflage de l'origine juive du fondateur de la famille. En effet, l'indianité se construit ici par l'ancrage territorial dans une « terre d'Indiens» (terra dos índios) sur plusieurs générations. Les récits insistent sur l'appauvrissement progressif, au fil des partages successoraux, des descendants de Luiz Tupaiu et sur un mode de vie similaire à celui attribué aux « índios ». Le surnom Tupaiu de l'aïeul de référence témoigne du passage du statut de descendants d'un immigré juif à celui de «natifs » (nativos). Cet ancrage graduel dans l'indianité est également perçu du point de vue du mélange biologique. En pratiquant des unions exogames, les Tapajós de l'Arapiuns s'inscrivent biologiquement et culturellement en Amazonie.

Que signifie alors être descendant de Juif dans le bas-Amazone ? L'héritage juif, pour les descendants de Luiz Caetano Tapajós, est conçu comme la transmission d'un patrimoine génétique (peau, yeux et cheveux clairs). Au sein des villages indigènes de l'Arapiuns, où l'exogamie est de mise, la transmission de caractéristiques physiques associées à la judaïté est plus aléatoire que dans la plaine d'inondation. Cependant, elles sont toujours valorisées. Les groupes résidentiels constitués par les parentèles Tapajós-Tupaiu continuent à être considérés comme des «grands blancs» par les autres familles. Ce statut, transposé du Jary vers l'Arapiuns, leur permet d'entretenir et d'alimenter de la différenciation sociale à l'intérieur des villages vis-à-vis d'autres habitants aux origines africaines ou amérindiennes. La hiérarchisation repose ainsi, du moins en partie, sur l'apparence physique et les origines du migrant fondateur.

\section{Discussion conclusive}

Ces deux études de cas retracent la trajectoire spatiale, généalogique et sociale d'hommes juifs immigrés au Maroc et en Amazonie. Dans les deux cas, on observe un même phénomène de captation de l'étranger et de ses compétences dans les lignages locaux. Puis, des stratégies sont mises en œuvre afin de l'autochtoniser, permettant sa permanence au sein du groupe d'accueil à plus long terme, en tant que fondateur de lignée, avec les singularités qui lui sont

\footnotetext{
${ }^{8}$ La Constitution de 1988 prévoit la démarcation de Terres Indigènes pour les indígenas. Ces-derniers sont reconnus par auto-identification depuis la ratification de la Convention n ${ }^{\circ} 169$ de l'Organisation internationale du travail, en 2003.
} 
propres. Dans l'exemple marocain, les compétences prisées chez le Juif Ali sont d'ordre relationnel et ésotérique : habile magicien et orateur, il charme sa parentèle d'adoption et les groupes avoisinants jusqu'à devenir un personnage charismatique. Dans l'exemple amazonien, ce sont les attributs physiques du Juif Lévi et son sens des affaires qui sont valorisés. Dans les deux cas, l'intégration passe par différentes étapes de re-nomination, une conversion religieuse et le mariage avec une épouse locale qui lui assure une descendance et l'accès à des terres. Prenant le nom d'une figue, dans le cas marocain, ou d'un fleuve, dans le cas Amazonien, l'origine exogène se teinte d'entrée d'une connotation locale pour mieux se faire adopter.

Ces deux exemples ont pour originalité de se trouver à mi-chemin entre le paradigme nouveau-chrétien du déni des origines et son opposé, celui de la reconstruction ailleurs d'un judaïsme confessionnel. Ici, le judaïsme est savamment mélangé (généalogiquement et religieusement) de façon à créer une autochtonie riche de singularités permettant de cultiver la différence, voire, de reproduire de la différenciation au sein de populations régionale que l'on pourrait à tort considérer comme des groupes sociaux homogènes. Ainsi, dans nos exemples, l'origine juive n'est ni conservée ni effacée. La filiation juive peut être soit entretenue par les générations descendantes grâce à des pratiques endogames qui s'inscrivent dans des stratégies de conservation de l'indivision de la terre ou au contraire être considérée comme un nouvel apport aux pratiques exogamiques. D'ailleurs, cet apport d'origine extérieure - l'immigré, le banni - est valorisé : dans le cas marocain, il est évoqué par la rumeur collective pour relativiser le caractère purement endogène et patrilinéaire de la filiation chérifienne ; en Amazonie, il est revendiqué et participe à une multiplicité d'origines motrice d'autochtonie.

Mettre en regard ces deux exemples a aussi pour intérêt d'éclairer la dynamique de l'origine exogène dans les trajectoires historiques de mondialisation comme celle impulsée par la construction des Etats empires ibériques. Ces deux exemples illustrent deux parcours de vie de l'origine juive dans un réseau migratoire qui fonctionne en vase communiquant entre le Portugal, le Maroc et l'Amazonie. Cette trajectoire a été notamment suivie par de nombreux Juifs séfarades tout au long de l'histoire, comme c'est le cas des ascendants du Juif Ali du Portugal à Ouled Ajar, et du Juif Lévi de Mogador à Arapixuna. Ces exemples nous montrent ainsi combien les phénomènes migratoires - juifs notamment - peuvent être lus comme différentes strates qui se superposent les unes aux autres. Ils nous rappellent que les origines sont toujours multiples, et que la catégorisation entre l'étranger et l'autochtone n'est pas toujours aussi évidente qu'on pourrait le penser. Ainsi, de la même façon que nos enquêtes questionnent la définition traditionnelle de l'autochtonie (ici l'étranger en est un moteur), elles 
proposent une réflexion sur ce que peut signifier être d'origine juive dans les territoires marqués par le contexte historique de la construction des Etats empires espagnols et portugais.

\section{Références}

Benchimol, S. 2008, Eretz Amazônia. Os Judeus na Amazônia, 3 ed., Manaus, Valer editora.

Berque, J. 1955, Structures sociales du Haut-Atlas, Paris, Presses universitaires de France.

Boyer, V. 2015, «Énoncer une “identité” pour sortir de l'invisibilité. La circulation des populations entre les catégories légales (Brésil)», L'Homme 2(214) : 7-36.

Coudreau, H. A. 1886, La France équinoxiale, études sur les Guyanes et l'Amazonie. Paris, Challamel.

Gellner, E. 2003, Les Saints de l'Atlas, Trad. Paul Coatalen, Saint-Denis, Bouchène.

Ibn Haldūn, 'A. al-R. ibn M. 1967, Discours sur l'histoire universelle, Trad. Vincent-Mansour Monteil, 3 vol, Beyrouth, Commission libanaise pour la traduction des chefs-d'œuvre.

Jacques-Meunié, D. 1982, Le Maroc saharien des origines au XVIe siècle, Paris, Klincksieck.

Jamous, R. 1981, Honneur et baraka : les structures sociales traditionnelles dans le Rif, Cambridge, Cambridge University Press.

Kenbib, M. 1994, Juifs et musulmans au Maroc : 1859-1948, Rabat, Université Mohammed V.

Le Cointe, P. 1922, L'Amazonie brésilienne : le pays, ses habitants, ses ressources, notes et statistiques jusqu'en 1920, vol. 1, Paris, A. Challamel.

Levy, A. 2003 "Notes on Jewish-Muslim Relationships: Revisiting the Vanishing Moroccan Jewish Community", Cultural Anthropology, 18 (3) : 365-397.

Nugent, S. 2009, "Some other Amazonians. Jewish Communities in the Lower Amazon". in S. Nugent \& M. Harris eds., Some Other Amazonians. Perspectives on Modern Amazonia, London, Institute for the Study of the Americas: 104-117.

Rozeaux, S. 2014, "Les horizons troubles de la politique de «colonisation» au Brésil: réflexions sur l'identité de la nation brésilienne à travers le prisme de la question migratoire (1850-1889)", Espace populations sociétés. Space populations societies [en ligne], 2-3. Consulté le 2 janvier 2019. http://eps.revues.org/5743

Sebti, A. 1992, "Insécurité et figures de la protection au XIXe siècle. La «ztâta » et son vocabulaire" in N. El Aoufi ed., La société civile au Maroc, Rabat, SMER : 24-29.

Simenel, R. 2010, L'origine est aux frontières. Les Aït Ba'amran, un exil en terre d'arganiers (Sud Maroc), Paris, Éditions de la Maison des sciences de l'homme.

Stoll, E., Fischer, L. Rodrigues da Cunha, Folhes, R. Theophilo 2017, "Recenser la propriété en Amazonie brésilienne au tournant du xxe siècle. Des registres de terres de João de Palma Muniz aux cartes de Paul Le Cointe”, Histoire \& mesure, XXXII(1) : 53-90. 


\section{Version pré-print}

Tavares, R. L. 1876, O Rio Tapajoz. Memoria onde se estenda semelhante tributario do Amazonas, não so como elemento de riqueza e uma das melhores vias de communicação, como tambem porque todo o territorio que banha é o mais apropriado para o estabelecimento de colonias agricolas e industriaes, Rio de Janeiro, Typographia Nacional.

Vidal, L., 2005, Mazagão. La ville qui traversa l'Atlantique, Paris, Flammarion.

Wachtel, N. 2001, La foi du souvenir : labyrinthes marranes, Paris, Seuil.

Wachtel, N. 2011 Mémoires marranes : itinéraires dans le sertão du Nordeste brésilien. La librairie du XXIe siècle, Paris, Seuil.

\section{Sources}

\section{Documents non-publiés}

Pinto, M. « Arapixuna - sua história ». Manuscrit dactylographié sans date.

Stoll, E. 2014, Rivalités riveraines : territoire, stratégies familiales et sorcellerie en Amazonie (Brésil), thèse de doctorat en anthropologie, Paris, EPHE \& Belém, UFPA.

\section{Archives}

Archive familiale Zabun, Acte de rahn (antichrèse). 1941, Mirleft.

Archive familiale Zabun, Généalogie du saint Sidi M'hend ou Yussuf. 1959, Mirleft.

Paroquia de Santarém, Registro de casamentos (Santarém, Vila Franca), 1888 - 1943.

Paroquia de Santarém, Livros de baptismos (Santarém, Vila Franca, Vila Curuai), 1872 - 1919.

Tribunal de Justiça do Estado do Pará. Juizo da Provedoria da Comarca de Santarém, Estado Confederado do Pará. Autos cíveis de inventário dos bens que ficaram por falecimento de Luiz Caetano Tapajós em que é inventariante seu filho Jayme Caetano Tapajós, 1894.

Tribunal de Justiça do Estado do Pará. Juizo de Direito da Comarca de Santarém, Estado Confederado do Pará. Autos cíveis de testamento em que é testador Luiz Caetano Tapajós, 1894. 